\title{
Freqüência e critérios para indicar a episiotomia
}

FREQUENCY AND CRITERIAFOR THE INDICATION OF EPISIOTOMY

FRECUENCIA Y CRITERIOS PARA INDICAR LAEPISIOTOMÍA

\author{
Sonia Maria Junqueira V. de Oliveira ${ }^{1}$, Elaine Cristina Miquilini ${ }^{2}$
}

\begin{abstract}
RESUMO
Trata-se de estudo exploratório com o objetivo de identificar a freqüência, os tipos e os critérios adotados para indicar a episiotomia. Foram entrevistados 12 médicos e 12 enfermeiras que prestam assistência à parturiente no Hospital Universitário da Universidade de São Paulo. A episiotomia ocorreu em 76,2\% dos partos normais; as indicações mais freqüentes foram: rigidez perineal $(28,7 \%)$, primiparidade $(23,7 \%)$, feto macrossômico $(11,9 \%)$, prematuridade $(10,2 \%)$. $\mathrm{O}$ tipo mais citado foi médiolateral direito (92,0\%), justificado por: aprendizado durante a formação acadêmica $(25,9 \%)$, ser adotada rotineiramente $(19,4 \%)$, menor chance de lesar o esfíncter anal $(16,1 \%)$, menor risco de complicações $(16,1 \%)$. É necessário rever as práticas de atendimento à parturiente, considerando as evidências científicas e condutas individualizadas.
\end{abstract}

\section{ABSTRACT}

This is an exploratory study aimed at identifying the frequency, the types and the criteria adopted to recommend episiotomy. Twelve doctors and 12 nurses who attend women giving birth at the University of São Paulo's Hospital Universitário were interviewed. Episiotomy was performed in 76.2 percent of the normal births; the most frequent indications were: perineal rigidity (28.7 percent), primiparity (23.7 percent), macrossomic infant (11.9 percent) and prematurity (10.2 percent). The most mentioned type was the right medium-lateral (92.0 percent), and the justifications were: it was learned during academic formation (25.9 percent); it is adopted routinely (19.4 percent); with it there is a lesser chance for causing lesions to the anal sphincter (16.1 percent); with it there is a lesser risk of complications (16.1 percent). The practices for attending women giving birth must be revised taking into account scientific evidences and individualized conducts.

\section{DESCRITORES}

Episiotomia.

Parto normal.

Períneo.

\section{KEY WORDS}

Episiotomy.

Natural childbirth.

Perineum.

\section{RESUMEN}

Trata-se de estudo exploratório com o objetivo de identificar a freqüência, os tipos e os critérios adotados para indicar a episiotomia. Foram entrevistados 12 médicos e 12 enfermeiras que prestam assistência à parturiente no Hospital Universitário da Universidade de São Paulo. A episiotomia ocorreu em $76,2 \%$ dos partos normais; as indicações mais freqüentes foram: rigidez perineal $(28,7 \%)$, primiparidade $(23,7 \%)$, feto macrossômico $(11,9 \%)$, prematuridade $(10,2 \%)$. O tipo mais citado foi médiolateral direito $(92,0 \%)$, justificado por: aprendizado durante a formação acadêmica $(25,9 \%)$, ser adotada rotineiramente $(19,4 \%)$, menor chance de lesar o esfíncter anal $(16,1 \%)$, menor risco de complicações $(16,1 \%)$. É necessário rever as práticas de atendimento à parturiente, considerando as evidências científicas e condutas individualizadas.

\section{DESCRIPTORES}

Episiotomia. Parto normal. Períneo. 


\section{INTRODUÇÃO}

A partir do século XVIII, houve um crescente interesse da medicina pela reprodução, e a entrada dos homens no quarto de parir coincidiu com a incorporação da prática obstétrica, cirúrgica, à medicina. Esse fato trouxe um aumento da mortalidade das mães e crianças, provocada sobretudo pela febre puerperal, pelo uso de instrumentos e pela prática da cesárea ${ }^{(1-2)}$. Assim, o parto foi adquirindo o caráter patológico, sendo necessário para seu manejo a hospitalização da mulher.

Atualmente, a episiotomia é um dos procedimentos mais comuns em obstetrícia, sendo superado apenas pelo corte e pinçamento do cordão umbilical $^{(2)}$. A episiotomia foi sugerida para auxiliar os partos vaginais complicados. No entanto, começou a ser recomendada sistematicamente por dois eminentes ginecologistas, sendo o primeiro deles Pomeroy, que em 1918 escreveu um artigo intitulado "Deveríamos cortar e reparar o períneo de todas as primíparas?". Em 1920, De Lee sugere a utilização do fórcipe profilático acompanhado de uma episiotomia médio-lateral precoce ${ }^{(3)}$.

Entre 1915 e 1925, a episiotomia era usada de modo profilático, como justificativa para evitar traumas perineais e prevenir a morbimortalidade infantil e problemas ginecológicos, tais como retocele, cistocele e relaxamento da musculatura pélvica ${ }^{(4)}$. Esse modelo vem sendo adotado e ensinado pela obstetrícia brasileira como uma das muitas condutas bem estabelecidas e universalmente aceitas; sendo justificado pelos autores para prevenir o suposto afrouxamento pélvico irreversível do parto. Sabe-se que o tônus da musculatura da vagina depende mais do exercício e da consciência da mulher, de sua contração e do relaxamento, do que de cirurgias de rotina ${ }^{(5)}$.

A justificativa habitual para o uso da episiotomia inclui a prevenção do trauma perineal severo, de danos do assoalho pélvico, de prolapso e de incontinência urinária. No entanto, apesar do uso largamente difundido, não há evidências científicas que suportam esses benefícios ${ }^{(6-8)}$.

Episiotomia é uma incisão cirúrgica na região da vulva, com indicação obstétrica para impedir ou diminuir o trauma dos tecidos do canal do parto, favorecer a liberação do concepto e evitar lesões desnecessárias do pólo cefálico submetido à pressão sofrida de encontro ao períneo ${ }^{(9)}$. A incisão costuma ser feita quando a cabeça fetal está suficientemente baixa, a ponto de distender o períneo, porém, antes de ocorrer uma distensão exagerada.
Também não pode ser realizada cedo demais, pois, deve-se prevenir um sangramento excessivo ${ }^{(10)}$.

A episiotomia pode ser lateral, médio-lateral e mediana, neste caso denominada de perineotomia. A episiotomia lateral está abandonada por seus inconvenientes, pois essa região, além de muito vascularizada, ainda pode lesar os feixes internos do músculo elevador do ânus. A episiotomia médio-lateral é a mais usada, e a incisão abrange pele, mucosa vaginal, aponeurose superficial do períneo e fibras dos músculos bulbocavernoso e do transverso superficial do períneo e algumas vezes, fibras internas do elevador do ânus. A episiotomia mediana apresenta como vantagens menor perda sangüínea, é fácil de reparar, maior respeito à integridade anatômica do assoalho muscular, menor desconforto doloroso e raramente é acompanhada de dispareunia ${ }^{(10-11)}$.

Admite-se que a episiotomia, além de provocar maior perda sangüínea, não previne posteriores transtornos do assoalho pélvico, podendo em alguns casos aumentá-los ${ }^{(2)}$. Estudo com 2.188 partos consecutivos, na Dinamarca, classificou as parteiras de acordo com suas taxas de episiotomias: parteiras com baixas taxas $(22,0 \%)$, parteiras com taxas médias $(40,0 \%)$ e parteiras com altas taxas $(55,0 \%)$, sendo que as mulheres assistidas por parteiras com baixas taxas conservavam com maior freqüência o períneo íntegro $(37,5 \%$ contra $25,5 \%$ dos demais grupos). Um fator importante a ser mencionado é que cerca de $45,0 \%$ das mulheres eram primíparas $^{(12)}$.

Analisando-se os dados sobre os partos de nulíparas, constatou-se que lacerações profundas do períneo ocorreram em maior número nas mulheres que foram submetidas à episiotomia, concluindo que o uso seletivo de episiotomia poderia minimizar os traumas dos partos normais em nulíparas $^{(13)}$. Até onde se tem conhecimento das seqüelas da episiotomia, a longo prazo, como incontinência urinária e dispareunia, independem do uso restrito ou liberal do procedimento ${ }^{(2)}$. Mulheres que tiveram episiotomia de rotina apresentaram piora na função sexual em relação àquelas que não foram submetidas ao procedimento, portanto, não há evidência que o uso rotineiro de episiotomia tenha efeito benéfico ${ }^{(14)}$.

Outro fator que aumenta a taxa de episiotomia é manter a mulher deitada, uma vez que implica no aumento da duração do trabalho de parto e do risco de sofrimento fetal, pela diminuição da intensidade e da eficácia das contrações. Na posição dorsal horizontal, há pressão da veia cava inferior, que diminui o fluxo sangüíneo de oxigênio para o
Freqüência e critérios para indicar a episiotomia 
Sonia Maria J.V. de Oliveira Elaine Cristina Miquilini feto. Como é mais desconfortável ficar deitada, a tendência é o aumento da tensão e da dor, o que estimula a solicitação da anestesia ${ }^{(15)}$.

Em estudo prospectivo de 2.144 partos realizados na Suécia encontrou-se taxa de infecção significativamente mais elevada $(p<0,001)$ no grupo de mulheres submetidas à episiotomia $(10,2 \%)$, comparadas às que tiveram laceração espontânea $(2,4 \%)$ e que nos últimos anos, alguns autores têm questionado o uso rotineiro da episiotomia ${ }^{(16)}$. Após análise de 356 partos de prímiparas e 341 de multíparas, concluíram que a episiotomia deveria ser adotada com restrição, apenas nos casos onde houvesse indicações específicas para mãe e feto ${ }^{(7)}$.

Em 1996, peritos em obstetrícia da Organização Mundial da Saúde (OMS) publicaram o "Guia Prático para Assistência ao Parto Normal" com base em 218 estudos, que apontam as melhores evidências científicas, classificaram as práticas relacionadas ao parto normal em quatro categorias:

Categoria A: Práticas demonstradamente úteis e que devem ser estimuladas;

Categoria B: Práticas claramente prejudiciais ou ineficazes e que devem ser eliminadas;

Categoria C: Práticas em relação às quais não existem evidências suficientes para apoiar uma recomendação clara e que devem ser utilizadas com cautela, até que mais pesquisas esclareçam a questão;

Categoria D: Práticas freqüentemente utilizadas de modo inadequado. O uso liberal ou rotineiro da episiotomia está classificado na Categoria $\mathbf{D}^{(17)}$.

Essa publicação afirma ainda que

não existem evidências confiáveis que o uso liberal ou rotineiro da episiotomia tenha um efeito benéfico, mas há evidências claras de que pode causar dano. Num parto, até então normal, pode ocasionalmente haver uma indicação válida para uma episiotomia, mas recomenda-se o uso limitado dessa intervenção.

Complementa que os sinais de sofrimento fetal, a progressão insuficiente do parto e a ameaça de laceração de $3^{\circ}$ grau, incluindo laceração de $3^{\circ}$ grau em parto anterior, são indicações para realizar a episiotomia. A ocorrência de lacerações perineais é freqüente em partos normais, porém lacerações de $1^{\circ}$ grau às vezes não necessitam de sutura, enquanto lacerações de $2^{\circ}$ grau podem ser suturadas e, em geral, cicatrizam sem complicações. Já as lacerações de $3^{\circ}$ grau podem ter conseqüências mais sérias com problemas de fístulas ou incontinência fecal ${ }^{(17)}$.

Muitos estudos têm avaliado as práticas e técnicas relativas ao período expulsivo, com o objetivo de diminuir as intervenções desnecessárias e a morbimortalidade materno-fetal. Nesse sentido, atuando na assistência à parturiente, sentimos necessidade de realizar um estudo para conhecer as indicações e os fatores que influenciam na decisão dos profissionais para realizar a episiotomia.

\section{OBJETIVOS}

- Verificar a freqüência de episiotomia nos partos espontâneos;

- Identificar os critérios adotados para indicar a episiotomia;

- Identificar quais os tipos de episiotomia adotados.

\section{METODOLOGIA}

\section{Tipo de estudo}

É um estudo quantitativo, descritivo e transversal.

\section{Local}

Este estudo foi desenvolvido no Hospital Universitário da Universidade de São Paulo (HUUSP).

\section{População}

A população foi constituída por médicos obstetras e enfermeiras obstétricas que prestam assistência à parturiente no Centro Obstétrico $(\mathrm{CO})$, o qual conta com 12 enfermeiras obstétricas e 29 médicos. O total de enfermeiras que participaram deste estudo, foi igual ao número de médicos, os quais foram selecionados por amostragem acidental.

\section{Procedimento de coleta de dados}

Foi consultado o livro de registro dos partos no $\mathrm{CO}$, para verificar a freqüência de rotura, integridade perineal e realização da episiotomia nos partos normais, durante o mês de outubro de 2000. Para esta pesquisa foi utilizado um questionário semi-estruturado composto de questões abertas e fechadas. A coleta de dados teve início após ser obtida a permissão para realizar o estu- 
do junto às comissões de ética e pesquisa do HU-USP. Uma das pesquisadoras fez contato com as 12 enfermeiras e 12 médicos, em seus respectivos turnos de trabalho e a entrevista só ocorreu após a assinatura do documento "consentimento livre e esclarecido para participar de pesquisa científica". Também foi garantido o anonimato dos participantes.

\section{Tratamento dos dados}

Os resultados, apresentados na forma de gráficos e tabelas, foram analisados em função de sua freqüência absoluta e índices percentuais. As questões abertas foram agrupadas e classificadas, segundo o conteúdo e analisadas de forma descritiva.

\section{RESULTADOS E DISCUSSÃO}

Os dados apontam que dos 255 partos ocorridos no mês de outubro de 2000, 47,8\% foram partos normais, $36,9 \%$ cesáreas e $15,3 \%$ fórcipes. Do total de 122 partos normais, em $76,2 \%$ foram realizadas EMLD, em 10,6\% o períneo manteve-se íntegro, em $9,0 \%$ ocorreram rotura de $1^{\circ}$ grau e $4,1 \%$ de $2^{\circ}$ grau, conforme mostra a Figura 1. Nesse período não houve nenhum caso de rotura de $3^{\circ}$ grau, que poderia ter conseqüências mais sérias para a mulher. Convém mencionar que dos partos normais $41,6 \%$ (42) eram de primigestas e constatou-se que a episiotomia foi praticada na maioria deles $(95,2 \%)$.

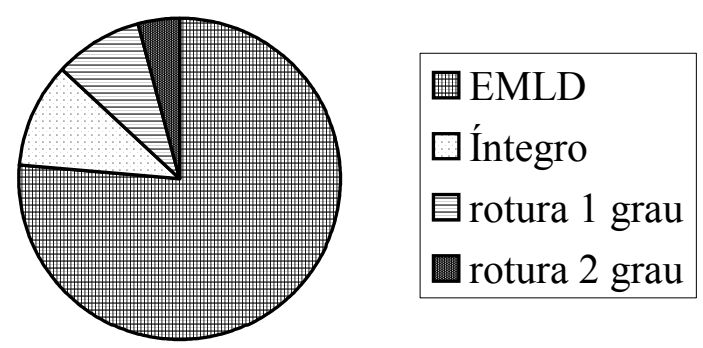

Figura 1. Condições perineais no parto normal. São Paulo, 2000.

De acordo com a publicação da OMS, lacerações de $1^{\circ}$ grau às vezes não necessitam de sutura, portanto, no atual estudo, dos partos que não foram realizadas episiotomia é de se supor que apenas nas roturas de $2 .{ }^{\circ}$ grau $(4,1 \%)$ haveria necessidade de sutura $^{(17)}$. A principal justificativa para adoção rotineira da episiotomia em todas as prímiparas é a prevenção de laceração perineal, de posterior relaxamento do assoalho pélvico e de trauma contra a cabeça fetal ${ }^{(18)}$. Essas autoras comentam que conseguiram reduzir a necessidade de episiotomia de um hospital-escola, mesmo em primíparas, adotando a deambulação livre durante o trabalho de parto.

Merece destacar que, se a episiotomia está indicada para todas as primíparas, assim como para as multíparas com episiotomia anterior, então esse procedimento acaba sendo praticado quase que em todos os partos, como rotina hospitalar, sem analisar outros fatores como vantagens e desvantagens para cada parturiente.

Um ensaio randomizado controlado realizado com 2.606 mulheres argentinas, no período de agosto de 1990 a julho de 1992, concluiu que o uso rotineiro de episiotomia deveria ser abandonado e taxas deste procedimento acima de $30 \%$ não podem ser justificadas ${ }^{(6)}$. A freqüência ideal de episiotomia, de acordo com os ensaios clínicos, deveria situar-se entre 10 e $30 \%$. Há dúvida quanto à taxa ideal, mas em muitos países está ocorrendo uma importante redução nas taxas de episiotomia ${ }^{(3)}$.

Tabela 1: Critérios para indicar a episiotomia no parto normal. (São Paulo, 2000)

\begin{tabular}{lrr}
\hline Critérios & N & \% \\
\hline Rigidez perineal & 17 & 28,7 \\
Primiparidade & 14 & 23,7 \\
Feto macrossômico & 7 & 11,9 \\
Prematuridade & 6 & 10,2 \\
Períneo íntegro & 4 & 6,8 \\
Episiotomia anterior & 3 & 5,1 \\
Apresentação pélvica & 2 & 3,4 \\
Períneo curto & 2 & 3,4 \\
Iminência de rotura & 2 & 3,4 \\
Outros & 2 & 3,4 \\
\hline Total & $59^{*}$ & 100 \\
\hline
\end{tabular}

* Cada profissional citou um ou mais critérios.

Conforme os dados da Tabela 1, o critério mais citado pelos médicos e enfermeiras obstétricas para realizarem esse procedimento foi a rigidez perineal $28,7 \%$ (17), seguido pela primiparidade $23,7 \%$ (14) e feto macrossômico $11,9 \%$ (7). A categoria rigidez perineal inclui citações como:

elasticidade do períneo, distensão perineal, condições do períneo, musculatura rígida, má distensibilidade e resistência perineal.

Esses resultados contrastam com os dados do estudo de outro estudo ${ }^{(19)}$ sobre sutura do trauma perineal no parto normal, que encontrou freqüência mais elevada de rigidez perineal
Freqüência e critérios para indicar a episiotomia 
Sonia Maria J.V. de Oliveira Elaine Cristina Miquilini
$(47,6 \%)$ e menores taxas de macrossomia fetal $(4,8 \%)$ e prematuridade $(2,4 \%)$ na indicação da episiotomia.

Resultados diferentes da presente pesquisa também foram observados ${ }^{(16)}$, constatando, entre as indicações mais citadas para realizar a episiotomia, a iminência de laceração e a asfixia fetal com $35,0 \%$ e $33,0 \%$ na primípara, $46,9 \%$ e $31,9 \%$ na multípara, respectivamente. Esses autores também verificaram que a episiotomia foi executada em $40,1 \%$ das primíparas e em apenas $9,9 \%$ das multíparas. Da mesma forma, em estudo feito com 186 parteiras, as indicações para realizar a episiotomia foram o sofrimento fetal $(63 \%)$, seguidos pela rotura iminente $(49 \%)$ e períneo rígido $(40 \%)^{(20)}$.

A justificativa para o fato de a episiotomia ser praticada, na maioria dos partos vaginais, pode ser a orientação dada nos livros textos de obstetrícia. Por exemplo, a obra de Jorge de Rezende que é amplamente utilizada pelos profissionais da área de obstetrícia e indica a episiotomia quando o períneo apresenta pouca elasticidade. Este autor $^{(9)}$ afirma que esse procedimento seria quase sempre indispensável nas primiparturientes e nas multíparas que já foram episiotomizadas. Esses dois últimos critérios também foram referidos pelos profissionais do atual estudo. Pode-se, pois, verificar que a maioria dos profissionais adotam os critérios transmitidos nos livros de obstetrícia.

Chama atenção que $6,8 \%$ dos profissionais citaram o períneo íntegro como critério de indicação da episiotomia, corroborando a afirmação anteriormente referida ${ }^{(9)}$. Nesse sentido, pode-se concluir que em todos partos normais haveria necessidade de indicar a episiotomia.

Outro critério apontado no presente estudo foi a questão do feto macrossômico. Na pesquisa sobre as taxas de episiotomia, rotura e integridade perineal em partos normais assistidos por enfermeiras obstétricas no Hospital Geral de Itapecirica da Serra, verificou-se que as maiores taxas de mulheres episiotomizadas foram daquelas que tiveram recém-nascidos $(\mathrm{RN})$ que pesavam entre 3.005 a 3.500 gramas $(37,6 \%)^{(22)}$. Das mulheres que mantiveram o períneo íntegro, $38,6 \%$ tiveram RN entre 3.005 e 3.500 gramas, $30,3 \%$ entre 2.505 e 3.000 gramas e $14,8 \%$ entre 3.505 e 4.000 gramas. Quanto às mulheres que apresentaram rotura de $1 .{ }^{\circ}$ grau $45,3 \%$ e de $2 .{ }^{0}$ grau, os RNs pesavam de 3.005 a 3.500 gramas. Concluí-se que, obedecendo a critérios individuais, não limitados à paridade, idade materna e peso do RN, é possível reduzir a taxa de episiotomia de rotina.

A prematuridade citada, por seis profissionais, como critério para executar a episiotomia, geralmente, é indicada como um procedimento que encurta o período expulsivo, evitando, desse modo, a compressão da cabeça fetal durante o trajeto pelo canal de parto. No entanto, não foi comprovado científicamente que realizar a episiotomia previne os traumas cranianos do concepto e os estudos que avaliaram a duração do período expulsivo relatam abreviação variando de um a nove minutos ${ }^{(3)}$.

$\mathrm{Na}$ categoria outros foram incluídas citações como evitar compressão cefálica e tempo disponivel na sala de parto.

Tabela 2 - Medidas adotadas pelos profissionais para proteger o períneo quando não é realizada a episiotomia. (São Paulo, 2000)

\begin{tabular}{|c|c|c|}
\hline Critérios & $\mathbf{N}$ & $\%$ \\
\hline Proteção/contenção do períneo & 19 & 46,4 \\
\hline Evitar desprendimento abrupto & & \\
\hline do pólo cefálico & 11 & 26,8 \\
\hline Massagear o períneo & 3 & 7,3 \\
\hline Lubrificar o períneo & 2 & 4,9 \\
\hline Orientar a mãe para moderar & & \\
\hline a força expulsiva & 2 & 4,9 \\
\hline Abaixar o períneo & 2 & 4,9 \\
\hline Aproximar a fúrcula & & \\
\hline no coroamento & 1 & 2,4 \\
\hline Evitar tracionar o feto & & \\
\hline durante o desprendimento & 1 & 2,4 \\
\hline Total & $59^{*}$ & 100 \\
\hline
\end{tabular}

* Cada profissional citou uma ou mais medidas.

As medidas mais citadas foram a proteção do períneo, 46,4\% (19), e evitar o desprendimento abrupto do pólo cefálico, 26,8\% (11). Na primeira categoria foram incluídas citações como utilização de compressa com leve compressão, proteção com as mãos, contenção e apoio perineal.

Segundo a publicação da OMS, muitos livros descrevem a prática de proteção do períneo durante o parto da seguinte forma: os dedos de uma das mãos do parteiro apoiam o períneo, enquanto a outra mão faz uma leve pressão sobre a cabeça para controlar a velocidade de coroamento, tentando evitar ou reduzir os danos perineais. Entretanto, afirma que é possível com essa mesma manobra evitar uma laceração de períneo, mas também pode ser que a pressão sobre a cabeça fetal impeça o seu movimento de extensão e a 
afaste do arco púbico em direção ao períneo, aumentando assim a possibilidade de lesão do períneo. Concluem que não há evidências suficientes sobre os benefícios desse método ${ }^{(17)}$.

Estudo experimental, randomizado e controlado, comparou a técnica intervencionista de proteção do períneo e a técnica expectante, com o objetivo de avaliar o trauma perineal ${ }^{(22)}$. Observou que não houve diferença estatística na freqüência de laceração do períneo em função da técnica usada. Conclui que a prevenção do trauma perineal pode estar mais associada à posição da mãe durante o parto, ao uso de ocitócicos, ao tipo de puxo e ao suporte emocional oferecido à parturiente do que à técnica de proteção do períneo.

Para melhor atender aos interesses maternos e perinatais, a liberação cefálica deve ser lenta e precedida de manobras que forcem ao máximo a flexão da cabeça, reduzindo a circunferência de desprendimento. Deve-se solicitar que a parturiente promova esforços expulsivos com a boca aberta, reduzindo assim a intensidade dos esforços, e permitindo o desprendimento final da apresentação de forma lenta ${ }^{(11)}$.

Outra medida de proteção do períneo referida pelos profissionais no presente estudo foi a massagem perineal, durante o final do período expulsivo, tentando relaxar os tecidos. Descrevese que a massagem pode ser feita por meio de movimentos circulares largos na vagina e de abaixamento, usando os dedos médio e indicador ${ }^{18}$. Os resultados de estudo prospectivo e randomizado demonstraram redução de $6,1 \%$ nas taxas de roturas de $2^{\circ}$ e $3^{\circ}$ graus e de episiotomias no grupo em que foi realizada massagem quando comparado com o grupo em que não foi feito o procedimento ${ }^{(23)}$.

Tabela 3 - Tipo de episiotomia adotada pelos profissionais. (São Paulo, 2000)

\begin{tabular}{lcc}
\hline Tipo & N & $\%$ \\
\hline EMLD & 23 & 92,0 \\
Mediana & 2 & 8,0 \\
\hline Total & $25^{*}$ & 100 \\
\hline
\end{tabular}

* Um profissional citou 2 tipos.

Em relação ao tipo de episiotomia adotado pelos profissionais , a maioria, 92\% (23) apontou a episiotomia médio-lateral direita (EMLD). A episiotomia do tipo mediana, também denominada perineotomia foi apontada por apenas dois profissionais, sendo que um deles indicou também a EMLD. Dados semelhantes a este estudo foram observados verificando-se que dentre os 410 par- tos ocorridos, a episiotomia realizada foi médiolateral, exceto em $14 \operatorname{casos}^{(16)}$.

A perineotomia respeita mais a anatomia da mulher quando comparada à episiotomia médiolateral, pois atinge apenas a pele, a mucosa vaginal e as fáscias superficiais e profundas do períneo, conforme explicação citada na Introdução ${ }^{(11)}$.

Tabela 4 - Justificativas apontadas pelos profissionais para adotarem a EMLD. (São Paulo, 2000)

\begin{tabular}{lcc}
\hline Justificativas & N & \% \\
\hline Aprendizado na formação acadêmica & 8 & 25,9 \\
Mais adotada rotineiramente & 6 & 19,4 \\
Menor chance de lesar esfíncter anal & 5 & 16,1 \\
Menor risco de complicações & 5 & 16,1 \\
Facilidade na sutura & 4 & 12,9 \\
Maior experiência & 2 & 6,4 \\
Períneo curto & 1 & 3,2 \\
\hline Total & $31^{*}$ & 100 \\
\hline
\end{tabular}

* Cada profissional apontou uma ou mais justificativas.

Pode-se observar, nos dados da Tabela 4, que grande número das justificativas $(64,6 \%)$ referidas para escolha da EMLD foram direcionadas às vantagens para os profissionais, tais como, a influência do aprendizado durante a formação acadêmica $(25,9 \%)$, mais adotada rotineiramente $(19,4 \%)$, facilidade na sutura $(12,9 \%)$ e maior experiência $(6,4 \%)$; as vantagens para as mulheres foram apontadas com menor freqüência $(35,4 \%)$, a saber, menor chance de lesar o esfíncter anal $(16,7 \%)$, menor risco de complicações $(13,3 \%)$ e períneo curto $(3,2 \%)$. Chama atenção a grande influência da formação acadêmica, apontada em um quarto das respostas. Considerando-se que o local de estudo é um hospital-escola, pode-se concluir que essa influência será repassada para as próximas turmas de formandos.

Dados semelhantes foram observados, encontrando-se maior dependência da educação profissional em oposição aos critérios médicos, dentre os fatores que influenciam a decisão de executar a episiotomia $^{(24)}$.

De acordo com os resultados do estudo feito em 2.172 partos, não houve a realização da episiotomia em 1.357 partos, no entanto, 14 dentre estes, apresentaram rotura de esfincter anal, e dos 815 com episiotomia médio-lateral, 25 tiveram lesão do esfincter anal. Portanto, mesmo sendo feita a episiotomia médio-lateral, as lesões do esfincter anal foram três vezes mais freqüentes em parturientes com episiotomia ${ }^{(12)}$.

Os dois profissionais que citaram o tipo mediano, apontaram como critérios de escolha a melhor
Freqüência e critérios para indicar a episiotomia 
Sonia Maria J.V. de Oliveira Elaine Cristina Miquilini cicatrização e reconstituição anatômica, além de menor chance de traumatismo, sangramento, deiscência, infecção e dor. Não obstante, um deles admite que, apesar de todos os pontos favoráveis à episiotomia mediana, costuma adotar a EMLD por hábito.

Segundo outro estudo ${ }^{(3)}$, a EMLD está associada a menor índice de lacerações de $3^{\circ} \mathrm{e} 4^{\circ}$ graus do que a episiotomia mediana, mas também é certeza que apresenta maior dificuldade técnica de reparação, é mais dolorosa no pós-parto e apresenta maior perda sangüínea. Esses autores recomendam que até que se obtenha estudos conclusivos, cada profissional deve escolher o método que considera mais adequado.

De acordo com nossos dados, a episiotomia ainda é um procedimento de rotina na maternidade estudada, apresentando altas taxas (76,3\%), índices superiores aos recomendados pela publicação da $\mathrm{OMS}^{(17)}$. Essa freqüência elevada de episiotomia pode estar relacionada com o fato do local do estudo ser um hospital-escola, portanto, os profissionais realizam esse procedimento com a finalidade de ensino. Outro fator que pode estar relacionado é o decúbito dorsal adotado pela parturiente durante o período expulsivo e o uso abusivo de indução do parto. Afirma-se que essa posição aumenta a tensão do períneo durante a passagem do feto pelo canal do parto ${ }^{(25)}$.

Por último, percebe-se a influência da formação acadêmica desses profissionais sobre o uso rotineiro da episiotomia. Culturalmente, não realizá-la é sinônimo de má assistência obstétrica no hospital' Comenta ter conseguido reduzir as taxas de episiotomia de $80 \%$ para $35 \%$, no Hospital Santa Marcelina, incorporando um programa de implementação de qualidade e educação continuada, com inauguração de suítes de partos que favorecem o parto natural e, além disso, liberando estatísticas e índices individualizados aos

\section{REFERÊNCIAS}

(1) Rezende J. Obstetrícia. 10. ${ }^{\mathrm{a}}$ ed. Rio de Janeiro: Guanabara Koogan; 2005. Obstetrícia: conceito, etimologia, propósitos, súmula histórica; p. 1-27.

(2) Osava RH. Assistência ao parto no Brasil: o lugar do não médico. [tese] São Paulo (SP): Faculdade de Saúde Pública USP; 1997.

(3) Ymayo MR, Bourget MM, Labrecque M, Eason E, Daniels F. Prevenção do trauma perineal no parto: porque evitar a episiotomia como rotina? São Paulo: s.n.; 1999. médicos e enfermeiros visando a sensibilização desses profissionais ${ }^{(26)}$

\section{CONSIDERAÇÕES FINAIS}

Com este estudo, foi possível observar que a episiotomia é realizada sem um enfoque seletivo. Atualmente, a taxa de episiotomia está diminuindo em todos os países, porém, devido à formação, por rotina e até mesmo os poucos estudos sobre o assunto, esse procedimento ainda prevalece em muitos hospitais como o local do presente estudo.

O uso rotineiro da episiotomia não está relacionado à redução da morbidade materna e fetal. É necessário um programa de educação continuada, mostrando aos profissionais estudos sobre as vantagens e desvantagens da episiotomia e formas de respeitar critérios individuais de indicação para o referido procedimento. Com base nas evidências científicas, a episiotomia está associada ao maior risco de laceração severa, logo, os profissionais devem buscar práticas alternativas para prevenir o trauma perineal, como posição lateral durante o expulsivo; puxo espontâneo, em contraposição ao dirigido; redução do uso indiscriminado de ocitocina e massagem perineal no final da gestação.

Outro fator que poderia contribuir para diminuir o índice de episiotomia seria um reajuste no olhar do profissional, isto é, ter como princípio que o parto normal é um processo fisiológico, devendo existir uma justificativa para interferir no trabalho de parto e nascimento, observando o paradigma não intervencionista. É necessário rever as práticas de atendimento à parturiente, considerando as evidências científicas e condutas individualizadas. Nesse sentido, há necessidade de estimular modelos de atendimentos mais humanizados, respeitando a singularidade de cada parturiente.
(4) Graham ID. Episiotomy: challenging obstetric interventions. London: Blackwell Science; 1997. The American crusade for prophylactic episiotomy; chap.3, p. 33-41.

(5) Diniz SG. Só um corte a mais? [editorial]. Genp Notas sobre nascimento e parto 1998; 3(6):1.

(6) Argentine Episiotomy Trial Collaborative Group. Rotine vs selective episiotomy: a randomized controlled trial. Lancet 1993; 342(8886-8887): 1517-8. 
(7) Klein MC, Gauthier RJ, Robbins JM, Kaczorowski J, Jorgensen SH, Franco ED et al. Relationship of episiotomy to perineal trauma and morbidity, sexual dysfunction, and pelvic floor relaxation. Am J Obstet Gynecol 1994; 171(3):591-8.

(8) Ecker JL, Tan WM, Bansal RK, Bishop JT, Kilpatrick SJ. Is there a benefit to episiotomy at operative vaginal delivery? Am J Obstet Gynecol 1997; 176(2):411-4.

(9) Rezende J. Obstetrícia. 10. ${ }^{\text {a }}$ ed. Rio de Janeiro: Guanabara Koogan; 2005. O Parto: estudo clínico e assistência; p. 336-62.

(10) Ziegel EE; Cranley MS. Enfermagem obstétrica. $8^{a}$ ed. Rio de Janeiro: Guanabara Koogan; 1985. Assistência da enfermagem durante o parto; p. $382-408$.

(11) Neme B. Ostetrícia básica. 2a ed. São Paulo: Sarvier; 2000. Parto: assistência; p. 190-213.

(12) Henriksen TB, Bek KM, Hedegaard M, Secher NJ. Episiotomy and perineal lesions in spontaneous vaginal deliveries. Br J Obstet Gynaecol 1992; 99(12):950-4.

(13) Borgatta L, Piening SL, Cohen WR. Association of episiotomy and delivery position with deep laceration during spontaneous delivery in nulliparous women. Am J Obstet Gynecol 1989; 160(2):294-7.

(14) Lede R, Belizan J, Carroll G. Is routine use of episiotomy justified? Am J Obstet Gynecol 1996; 174(5):1399-402.

(15) Diniz SG. O que funciona e o que é justo: notas sobre violência na assistência ao parto. In: Textos de apoio do $2 .{ }^{0}$ Seminário sobre Nascimento e Parto no Estado de São Paulo "Vitrine da Humanização em São Paulo"; 1999 maio 27-28; São Paulo. São Paulo: Secretaria de Estado de Saúde. Coordenação dos Institutos de Pesquisa; 1999.

(16) Larsson PG, Platz-Christensen JJ, Bergman B, Wallstersson G. Advantage or disadvantage of episiotomy compared with spontaneous perineal laceration. Gynecol Obstet Invest 1991; 31(4): 213-6.
(17) World Health Organization. Classification of practices in normal birth. In: Care in normal birth: a practical guide. Geneva; 1996. p. 34-7. (WHO Technical Report Series FRH/MSM/96.24)

(18) Osava RH, Tanaka ACA. Episiotomia de rotina: é realmente necessária? [Apresentado no Seminário de Enfermagem Obstétrica: a pesquisa na prática profissional; 1995 out 17-19; São Paulo].

(19) Silva SF. Sutura do trauma perineal no parto normal: estudo comparativo entre duas técnicas. [dissertação] São Paulo (SP): Escola de Enfermagem USP; 2002.

(20) Stamp SF. Care of the perineum in the second stage of labour: a study of views and practices of Australian midwives. Midwifery 1997; 13(2):100-4.

(21) Silva SF, Caroci AS, Riesco MLG, Basile ALO Ocorrência de episiotomia e rotura perineal no centro de parto normal do hospital geral de Itapecerica da Serra - SP. In: Resumo de trabalhos da $1^{\text {a }}$ Conferência Internacional sobre Humanização do Parto e Nascimento; 2000 nov. 2-4; Fortaleza. Fortaleza: JICA; 2000. p. 32.

(22) Costa ASC. Proteção perineal e manejo do feto no parto normal: estudo comparativo entre duas técnicas. [dissertação] São Paulo (SP): Escola de Enfermagem USP; 2002.

(23) Shipman MK, Boniface DR, Tefft ME, McCloghry F. Antenatal perineal massage and subsequent perineal outcomes: a randomized controlled trial. Br J Obstet Gynaecol 1997; 104(7):787-91.

(24) Gerrits DD, Brand R, Gravenhorst JB. The use of epesiotomy in relation to the professional education of the delivery attendent. J Obst Gynecol Reprod Biol 1994; 56(2):103-6.

(25) Abeche AM, Teruchkin B, Leite CSM, Mucenic M. Episiotomia: reavaliação de um procedimento clássico. Femina 1994; 22 (6/9):436-40.

(26) Ymayo MR. Mudanças na obstetrícia brasileira: alternativas para a redução dos índices de partos cesáreo e episiotomia em um hospital privado. In: Resumo de trabalhos da $1{ }^{a}$ Conferência Internacional sobre Humanização do Parto e Nascimento; 2000 nov 2-4; Fortaleza. Fortaleza: JICA, 2000. p. 29.
Freqüência e critérios para indicar a episiotomia
Correspondência: Sonia M.J.V. Oliveira Escola de Enferm da USP Av. Dr. Enéas de Carvalho Aguiar, 419 Cerqueira César - SP CEP -01440-902 - SP 